Renata L. Riha ${ }^{1,2}$

rlriha@hotmail.com

\title{
Review
}

\section{Defining obstructive sleep apnoea syndrome: a failure of semantic rules}

Obstructive sleep apnoea syndrome (OSAS) is one of the most ubiquitous medical conditions in industrialised society. Since the recognition that symptoms of excessive daytime somnolence, problems with concentration, mood and cognitive impairment, as well as cardiometabolic abnormalities can arise as a consequence of obstructed breathing during sleep, it has been subject to variation in its definition. Over the past five decades, attempts have been made to standardise the definitions and scoring criteria used for apnoeas and hypopnoea, which are the hallmarks of obstructive sleep apnoea (OSA). However, applying these definitions in clinical and research practice has resulted in over- and under-estimation of the severity and prevalence of OSAS. Furthermore, the definitions may eventually become redundant in the context of rapid technological advances in breathing measurement and other signal acquisition. Increased efforts towards precision medicine have led to a focus on the pathophysiology of obstructed breathing during sleep. However, the same degree of effort has not been focused on how and why the latter does or does not result in diurnal symptoms, integral to the definition of OSAS. This review focuses on OSAS in adults and discusses some of the difficulties with current definitions and the possible reasons behind them.

\section{Introduction}

Obstructive sleep apnoea (OSA) is a pathophysiological process characterised by obstruction of the upper airway during sleep, resulting in repetitive breathing pauses accompanied by oxygen desaturation and arousal from sleep. When this results in diurnal sleepiness, leads to cognitive impairment or cardiovascular morbidity it is called the obstructive sleep apnoea/ hypopnoea syndrome (OSAS), a disease [1]. Although several treatment modalities for OSAS have been developed over the past couple of decades, treatment is highly dependent on selfmanagement and most frequently comprises nightly use of either continuous positive airway pressure (CPAP) (considered the gold standard for all degrees of OSAS severity) or a mandibular repositioning device $[2,3]$.

That intermittent obstruction of the airway during sleep results in pathology and possible long-term harm has been recognised since the 1970s. Despite this recognition, the diagnosis and definition of OSAS continues to evolve. Factors that have played a major role in the evolution of OSAS definitions include: the sheer ubiquity of the condition and, thereby, the clinical load; rapid technological advances in diagnosis; recognition of the multiple endotypes and phenotypes of OSA that may have implications for management and
Cite as: Riha RL. Defining obstructive sleep apnoea syndrome: a failure of semantic rules. Breathe 2021 17: 210082. 
long-term health; and attempts at personalising treatment using strategies in addition to CPAP.

This review will focus on the definition of the disease OSAS in adults, i.e. the syndrome of excessive daytime sleepiness, cognitive impairment, and mood disturbance secondary to disordered breathing during the major sleep period (mostly at night). Sleep disordered breathing as a term per se comprises several sleep-related breathing pathologies which include snoring, OSA, OSAS, central sleep apnoea, hypoventilation syndromes and rarer forms of disturbed breathing. These will not be discussed.

Due to an inexplicable failure by the medical sleep community to adhere to and develop strict definitions of what OSAS is, and the sometimes arbitrary and nonevidence-based nature of what it comprises (in constant flux since the 1970s), there is significant conflation and interchangeable use of terms in the medical/scientific literature which is difficult to disentangle. Many studies focus purely on OSA (and its severity as measured by the apnoeahypopnoea index $(\mathrm{AHI})$ ), whilst others incorporate the daytime pathophysiological consequences into their definition and thereby investigate OSAS. This has resulted in a body of literature with conflicting results, the introduction of terms such as non-sleepy OSA and other, even more confusing, definitions.

\section{How do we define OSAS?}

Since it was the first recognised, definitions of OSAS have been derived from two components rated separately and very differently, namely the severity of OSA and daytime sleepiness. The objective metric of OSA is the AHI, which has itself been subject to a variety of definitions over the decades $[4,5]$.

Although the definition of OSAS seems relatively straightforward, i.e. the description of a pathophysiological process resulting in symptoms, even the International Classification of Sleep Disorders definitions have changed markedly over time (table 1) [6-8].

The third and current edition of the International Classification of Sleep Disorders (ICSD-3) [8], for instance, encompasses a very broad definition of OSAS (a synonym for OSA), defining it as: 1) clinical symptoms and complaints of diurnal sleepiness, or witnessed apnoeas by a partner, or cardiometabolic abnormalities and an $\mathrm{AHI}$ of $>5$ events per $\mathrm{h}$ of sleep; or 2) 15 or more predominantly obstructive breathing events per $h$ of sleep (with no attendant symptoms or comorbidities specifically listed). The implication of these definitions is that OSA should be treated since the daytime symptoms are being driven entirely and exclusively by the disturbed breathing during sleep. Although OSA is thought to affect approximately one billion people worldwide between the ages of 30 and 69 years [9], indiscriminate application of the ICSD-3 criteria [8] to data from large community-based cohorts would result in $50-70 \%$ of the population being defined as having OSAS [10]. Clearly, this is frightening, and unlikely to be the case.

The arbitrariness of the definition of OSAS and the need to discriminate it carefully from OSA can be illustrated from one of the most frequently cited epidemiological studies on its prevalence by Younc et al. [11]. In the Wisconsin Sleep Cohort Study, $24 \%$ of men $(n=325)$ and $9 \%$ of women $(n=250)$ had an $\mathrm{AHI}$ of $>5$ events per $\mathrm{h}$ of sleep [11]. However, when sleepiness was factored in as causally related to the $\mathrm{AHI}$, the prevalence fell to $4 \%$ in men and $2 \%$ in women. The reader is also reminded that the equipment used to acquire these data, and their scoring definitions have undergone profound changes since then. Finally, whether the reported sleepiness was directly attributable to the raised $\mathrm{AHI}$ was not validated. Several population prevalence studies since then have shown a mean (range) prevalence of OSA of $27.3 \%(9-86 \%)$ and $22.5 \%(3.7-63.7 \%)$ in men and women, respectively, with the mean prevalence of OSAS found to be $6 \%(3-18 \%)$ and $4 \%(1-17 \%)$ in men and women, respectively [12, 13]. Thus, OSA without self-reported sleepiness is almost three times as common in men and women as OSAS.

\section{Defining sleepiness}

According to the ICSD-3 [8], excessive daytime sleepiness is defined as the inability to stay awake and alert during the major waking episodes of the day, resulting in periods of irrepressible need for sleep or unintended lapses into drowsiness or sleep. Pathological sleepiness has been found to occur in $7-13 \%$ of the general population in large surveys and in $20-25 \%$ of a primary care population [14, 15]. The commonest cause of sleepiness in our society is behaviourally induced sleep insufficiency and men and women appear to be equally affected [16]. Furthermore, one review estimated that $42 \%$ of all sleepiness was likely to be secondary to a medical or psychiatric disorder rather than a sleep disorder per se [16]. However, up to $25-50 \%$ of OSAS patients do not report subjective tiredness or sleepiness [17], although they may be objectively and subjectively impaired in other ways (e.g. cognition is impaired). Objective definition of sleepiness is difficult but can be undertaken using a number of different behavioural measures including observation (e.g. yawning frequency, facial expression), performance tests (e.g. the driving simulator, psychomotor vigilance tests and reaction time tests), or direct electrophysiological measures (e.g. multiple sleep latency test (MSLT) and multiple wakefulness test, pupillometry and cerebral evoked potentials) [18].

Subjective measures include self-evaluation using rating scales (e.g. the Stanford Sleepiness Scale [19] to measure sleepiness at a given instant and the Epworth Sleepiness Scale (ESS) [20]. 


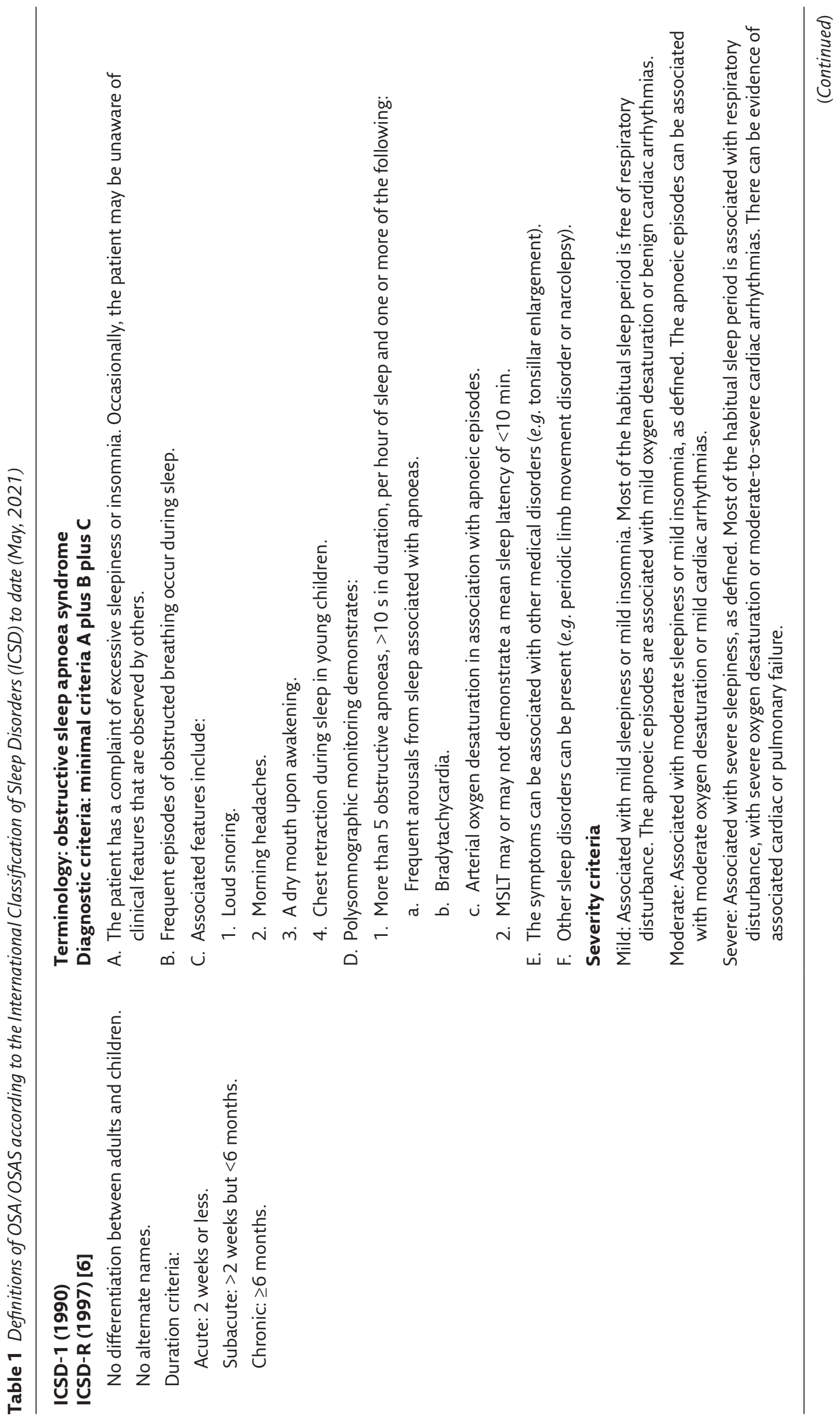




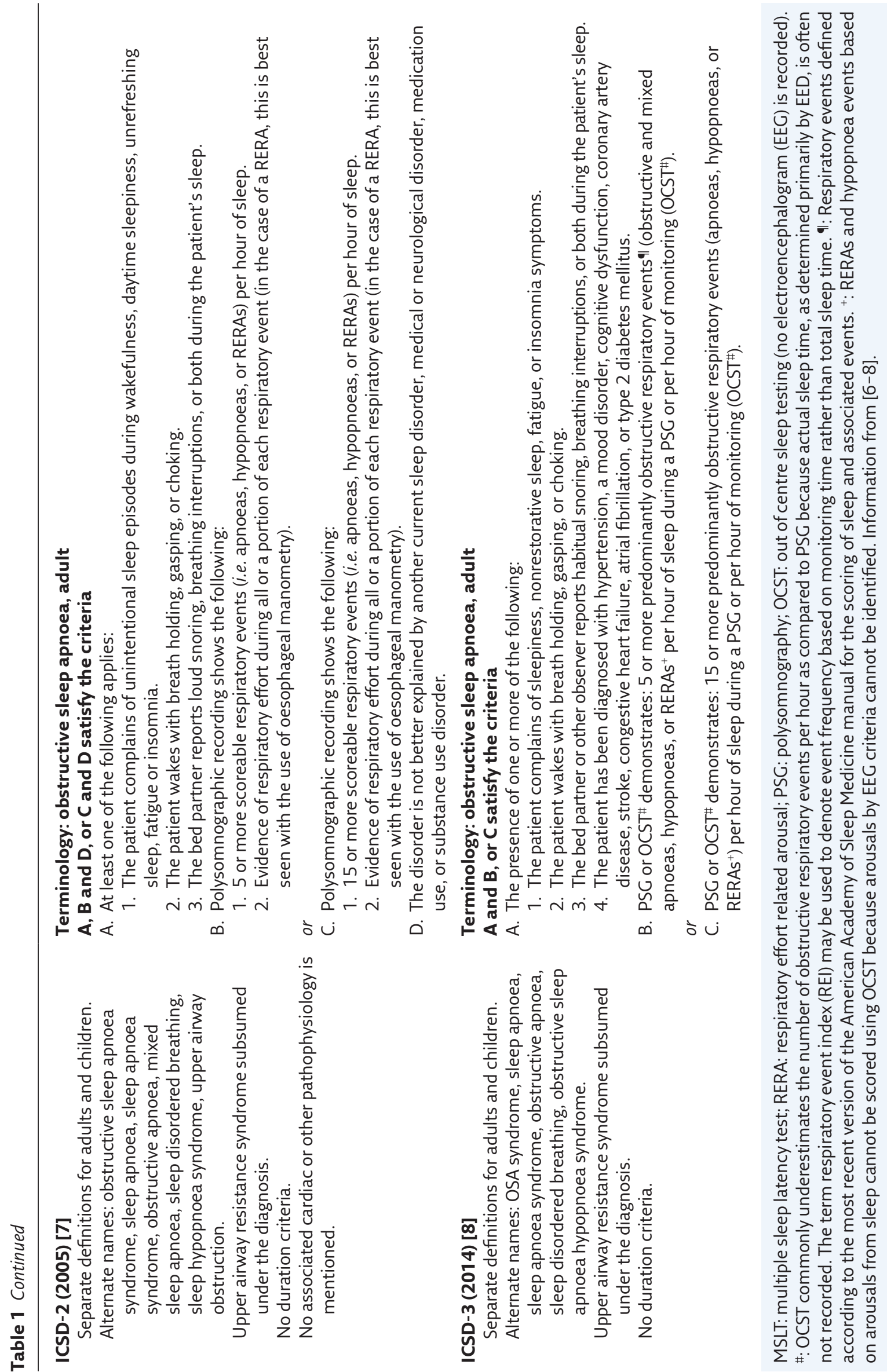


The most widely used scale internationally for assessing daytime sleepiness is the ESS [20]. An ESS of greater than 11 out of 24 (maximum score) is generally indicative of abnormal levels of daytime sleepiness, irrespective of age [12]. The ESS aims at measuring the general level of daytime sleepiness as a stable individual characteristic and has satisfactory test-retest reliability [20]. Clinically, the ESS is most frequently used to document intra-individual changes over time in a patient suspected of having OSAS to gauge some measure of subsequent treatment effectiveness. The ESS is used almost universally in research and clinical practice, not only in the diagnosis of OSAS but also to assess sleepiness per se in other sleep disorders and medical conditions that can results in sleepiness (e.g. narcolepsy, multiple sclerosis, traumatic brain injury). A pictorial version of the ESS (pESS) has also been developed and deployed in several studies [21]. Unfortunately, there are numerous significant limitations of the ESS and pESS, which include patient-minimisation of symptoms, misinterpretation, misunderstanding, and lack of relevance (e.g. someone who does not read may not even be able to imagine reading in one of the questions). Additionally, there are questionable intrinsic measurement properties of the ESS, as discussed in recent publications in the area [1, 22, 23]. Although an ESS of 11 out of 24 is considered pathological, there is evidence that there is a bell-curve distribution of scores within the non-OSAS population as there is in the OSAS population (figure 1) [24]. Medical and psychiatric comorbidities can result in daytime sleepiness and the more medical conditions the patient suffers from makes the ESS scores additive [25]. Talking to the patient, taking a collateral history and clinical observation are irreplaceable. Nevertheless, the ESS remains a useful tool clinically and for measuring sleepiness in large populations. A more refined questionnaire which is universally applicable is waiting to be developed!

Other questionnaires that have been used to screen in, screen out and increase diagnostic certainty of OSAS have also been used with varying degrees of success; their sensitivity and specificity depending on the population they have been trialled in. Screening questionnaires such as the STOP-Bang and its iterations [26], the Berlin Questionnaire [27] and NoSAS [28] are useful in screening for the presence or absence of the OSAS, but are currently neither universally adopted nor linked to for instance, economic evaluation algorithms as is the ESS [29, 30]. Finally, it is worth noting that subjective evaluation of sleepiness is open to error as people may willingly or unconsciously under or over-estimate their level of sleepiness.

Other methods of assessing daytime sleepiness objectively are not used in routine practice when it comes to diagnosing sleepiness in OSAS, but can be used to answer a specific clinical or medicolegal question (e.g. driving simulators [31], the SART

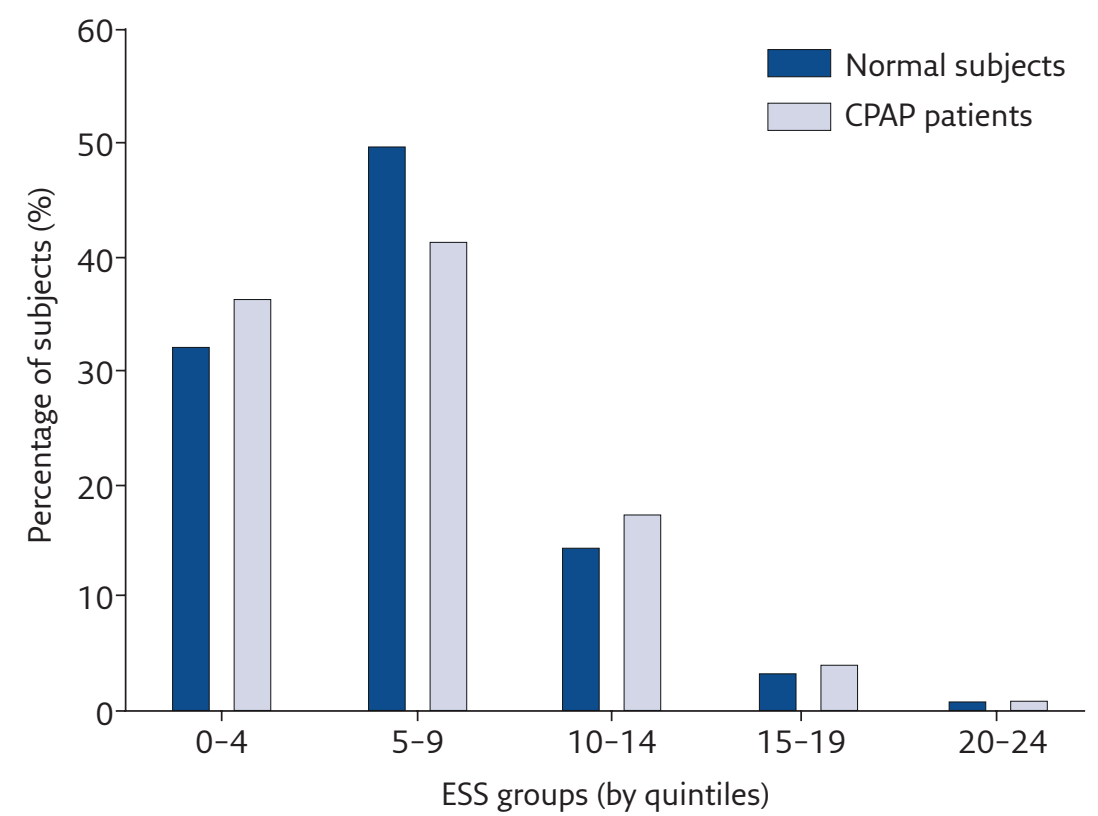

Figure 1 Distribution of ESS in randomly selected individuals from the community and patients on CPAP. Reproduced from [24] with permission.

test [32] and the OSLER test [33]), particularly if the diagnosis is unclear or another organic sleep disorder is suspected, e.g. narcolepsy. Probably the test most clinicians are most familiar with in respect of measuring sleepiness directly using an electroencephalogram (EEG) is the MSLT, which was originally designed to facilitate the diagnosis of narcolepsy [34]. The central premise of the MSLT is that the sleepier a subject is, the more quickly they will fall asleep. The maintenance of wakefulness test (MWT) is more frequently used in the context of OSAS, particularly when it comes to assessing driving safety [31]. The MWT measures the ability to stay awake for a defined period in a laboratorycontrolled, stimulus-free environment [34]. It is important to note, that despite their diagnostic usefulness under certain circumstances, no test of sleepiness can reliably predict sleepiness and performance in real-life situations with certainty.

\section{Defining OSA}

In addition to daytime sleepiness (objective or subjective), the definition of OSAS requires objective recording and measurement of OSA.

In decades past, the most widely used method for the diagnosis of OSA was polysomnography (PSG), and it is still considered the "gold standard" diagnostic instrument by which newer developments in the measurement of breathing during sleep continue to be assessed.

PSG is designed to simultaneously monitor the following: nasal and/or oral airflow; thoracoabdominal movement; snoring; EEG; electrooculogram (EOG); electromyogram (EMG); and oxygen saturation. The recording of any 
a)

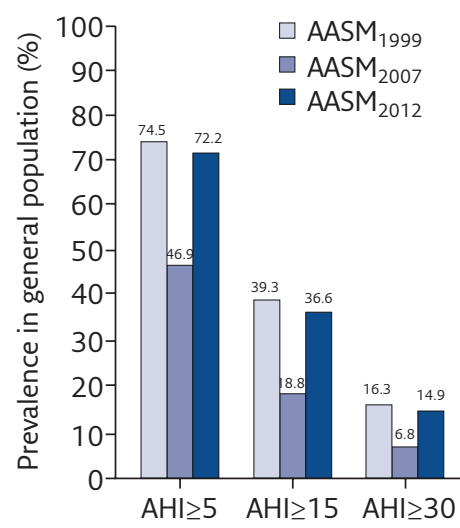

b)

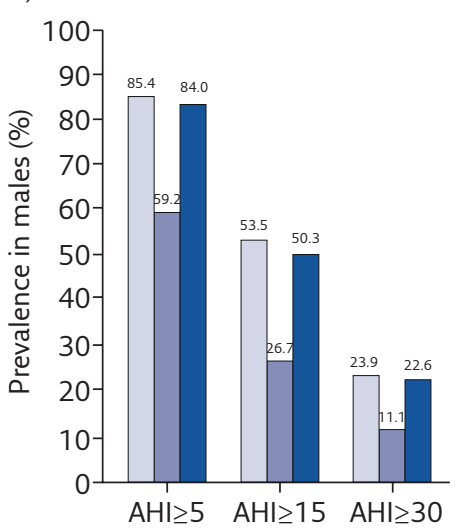

c)

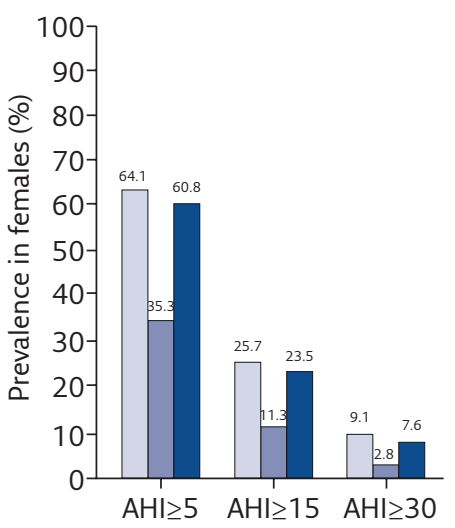

Figure 2 Differences in the prevalence of OSA in a cohort of patients in Lausanne, Switzerland, based on criterion C of the ICSD-3 (2014) definition according to different scoring criteria for hypopnoeas as mandated by the AASM scoring guidelines in 1999, 2007 and 2012. a) Whole cohort, b) males and c) females. Reproduced from [36] with permission.

abnormal movements using video may also help identify changes in airflow or desaturations. Signal collection and interpretation are now always computerised, but manual scoring of the trace must still be performed [35]. Numerous guidelines have been developed over the decades. Initially, scoring sleep and scoring respiratory events were subject to separate guidelines, but since 2007, all aspects of scoring the PSG have been integrated into one manual which is produced by the American Academy of Sleep Medicine (AASM) [35]. There have been fluctuations over the past 13 years in scoring rules for respiratory events which were shown to result in significant fluctuation in prevalence of OSAS (figure 2) and also on an individual level affected diagnosis [36]. Additional studies have demonstrated the shortcoming of the $\mathrm{AHI}$ as a metric: depending on the methods used, the numerical value of the $\mathrm{AHI}$ will differ [37, 38]. Finally, night-to-night variability of up to $20 \%$ in the number of breathing events recorded also affects prevalence and diagnosis [39].

Type III devices (synonyms: home sleep apnoea testing (HSAT), limited studies, polygraphy), can be used in documenting OSA using standalone cardiorespiratory monitoring [40]. This involves the measurement of airflow, respiratory effort, oxygen saturation and heart rate, but not EEG [35]. The great advantages of these systems are price, portability and convenience to patients who can set up the equipment in their own homes. The downside is the lack of EEG which can result in the over or usually under-scoring of respiratory events [41]. The difference between polygraphy and PSG can be over $20 \%$ which can have significant implications for accurate definition, hence diagnosis, management, outcomes, and reimbursement in different economic systems. Other systems are available that do not use flow limitation to document nocturnal sleep disordered breathing, e.g. the WatchPat ${ }^{\mathrm{TM}}$ device. However, they are still used in acquiring the signals incorporated in the definition and diagnosis of OSAS. Overnight oximetry alone is sometimes used as a screening test for identifying patients who are at risk of having significant OSA. However, oximetry should never be seen as a substitute for in-lab PSG or cardiorespiratory monitoring. The major limitation is the inability to detect apnoeas or hypopnoeas not associated with oxygen desaturation. Any nocturnal oxygen desaturation may also result from other disorders such as sleep hypoventilation without upper airway obstruction, e.g. COPD, severe kyphoscoliosis or muscular dystrophy [42]. The interpretation of oximetry traces can differ significantly among physicians calling into question the clinical utility of standalone overnight oximetry for defining OSA, although there might be a place for this in resource poor settings [43].

In 2011, Collop et al. [44] developed a classification system to evaluate the physiological information recorded by sensors in sleep diagnostic classification called SCOPER (sleep, cardiovascular, oxygen, position, effort, respiration). This system attempted to shift the paradigm of using separate sensors to a more physiologically based perspective, where it is recognised that with the evolution of technology, one sensor may deliver multiple bits of physiological information. This is important in the context of such a rapidly evolving field, where technological innovation has somewhat outrun our reference standards, including the "gold standard" PSG developed in the 1950s-1990s. New sensing systems may utilise and measure signals that we incorporate into current classification systems, yet which may turn out to be more useful with respect to diagnostic accuracy, hence definition, management, and outcome forecasting.

\section{Defining OSAS severity}

OSAS severity is not defined by the diurnal symptoms or degree of impairment in cognition, mood or ability to concentrate. Rather, it is defined by the $\mathrm{AHI}$ metric using PSG. The term $\mathrm{AHI}$ is used ubiquitously, 
although it should strictly not apply to any other method of acquiring it apart from PSG where sleep is documented. This includes type III devices and any other mode of measuring sleep disordered breathing which does not simultaneously record sleep using EEG. "Apnoeas", defined as a total cessation of airflow, but continued respiratory effort were initially identified as the pathological hallmark of OSA. Based on a limited number of studies and in limited populations, an arbitrary duration was set at $10 \mathrm{~s}$ minimum duration [5]. Later, the existence of "hypopnoeas" was recognised and incorporated into the definition of OSA/OSAS as equivalent in pathological outcomes (and both are now recognised as fundamental to the definition of OSA) [45]. Scoring criteria for respiratory events also underwent significant evolution over the years (figure 2 ). The choice of $3 \%$ and $4 \%$ desaturations, with or without arousals and decrements in breathing, as part of various hypopnoea definitions were also reached by consensus and based on expert opinion, rather than being evidence-based. Specifications of equipment used to acquire signals have not been standardised to date and very few studies in relation to this exist in the medical literature. With technological advances and digitisation of what was previously an analogue system, little consideration has been given to how this could modify scoring criteria over the years. Even the scoring of sleep using the EEG is based on the arbitrary imposition of a 30-s epoch on what is effectively continuous data which might be better assessed using other techniques such as spectral analysis.

The severity of OSA has been defined as either mild (5-15 events per $h$ of sleep), moderate (15-30 events per $h$ of sleep) or severe ( $>30$ events per $h$ of sleep) [46]. Again, these definitions, were introduced for research, rather than clinical purposes [5, 46] and are also purely arbitrary. Pevernagie et al. [5]. describe how in order to provide clinical guidance on when sleepiness could be attributable to OSA, a reference value was established to denote normalcy at $<5$ events per $h$ of sleep. This was based on a cohort of OSAS patients compared with 20 control subjects tested in the 1970s, gained favour with the majority of workers in the field at the time and has not been validated since [5]. Thus, the very definition of what constitutes OSA severity and normalcy of breathing during sleep has not been subjected to rigorous scrutiny. This is of concern as the $\mathrm{AHI}$ (and other metrics, such as the $\mathrm{A}+\mathrm{H} /$ time in bed for limited studies, respiratory disturbance index (RDI), respiratory event index (REI), etc.) with or without the presence of daytime symptoms is used to guide treatment decisions despite differences in acquisition, technological, and inter- and intra-scoring biases and variation within patient populations. Even predicting the risk of developing comorbidities or mortality risk will differ depending on how the $\mathrm{AHI}$ was acquired. Calls have been made to establish what might be considered normative data for the $\mathrm{AHI}$ according to age and sex in a specific population, but these have been largely ignored [1].

Recently, recognition of the shortcomings of the $\mathrm{AHI}$ as a metric to define OSA are leading workers in the field to consider alternatives, including hypoxic burden as measured by desaturation severity, arousal intensity, spectral analysis of sleep EEG, cardiopulmonary coupling, and apnoea-hypopnoea event duration. The reader is referred to an excellent overview of the subject by PEVERnAGIE et al. [5] and another recent discussion of the AHI by MALHOTRA et al. [4]. In summary, the $\mathrm{AHI}$ is not only a deficient metric, but is also a poor measure of clinical disease severity. No significant correlations have been demonstrated between $\mathrm{AHI}$ and sleepiness (both ESS and MSLT) [47], and furthermore, the AHI has not been shown to be predictive of associated comorbidities, e.g. prevalent hypertension [48], as discussed below. Thus, the AHI fails as a both a metric and a measure.

Leading on from this, how we define OSA and thus OSAS is particularly pertinent when we consider the proliferation of advanced technologies such as wearable technology, the use of artificial intelligence, data mining and machine learning, greater precision of blood biomarkers and improved genetic approaches. Among others, O'MAHONY et al. [49] have published a review of newer technologies being developed to measure OSA.

\section{Pathophysiology, endotypes and phenotypes of OSAS: importance to the definition}

The pathophysiology of OSAS has been briefly alluded to above. We know that OSAS is more common in men, in the obese, and is likely to present with greater severity in smokers and in the presence of alcohol [12]. OSAS presents somewhat differently in women, also in accordance with physiological life changes such as pregnancy and menopause [50]. OSAS runs in families and is probably due to heritable craniofacial and dental structures, control of breathing, and fat deposition propensity [12]. Craniofacial structure is extremely important as is nasal health and patency and lymphoid tissue overgrowth which can persist into adulthood or play a role subsequently in adulthood in determining severity of disease [51,52].

Apart from these anatomical features and risk factors, which contribute to the phenotype in the general adult population, the pathophysiological mechanisms that contribute to upper airway collapse resulting in apnoeas/hypopnoeas in OSA (endotypes) are currently postulated to be as follows: a smaller, more collapsible upper airway based on obesity and airway anatomy; reduced upper airway dilator muscle responsiveness; lower arousal threshold; and increased sensitivity of the ventilatory control system (loop gain) [53, 54]. A number of outstanding 
studies have been carried out examining these endotypes in patients presenting with OSA, with the development of classification systems in order to determine which treatment dispositions would be more suitable depending on the primary mechanism identified, e.g. PALM classification system [53]. Unfortunately, to date, no quick and easy method to deploy these findings clinically has been identified and some of them rely on what are considered cumbersome methods of investigation e.g. full PSG [55]. See figure 3 for an excellent summary of how phenotyping and endotyping patients with OSA might lead to improved definition of OSAS and, thus, greater treatment precision.

None of the approaches above have considered the endotype or phenotype for sleepiness secondary to the nocturnal disruption from arousals and intermittent hypoxaemia, so one could argue that this is currently a one-pronged approach, albeit one that has greatly increased our level of understanding of the pathophysiology of this complex condition.

Other methods of phenotyping patients with OSA and OSAS, and thus defining them, have been attempted by developing classification systems that automatically assume the presence of significant OSA but incorporate an additional main symptom, such as sleepiness or comorbidities. However, this does not necessarily mean that the symptom and the $\mathrm{AHI}$ are causally related and may not contribute to increasing the specificity of OSA as a clinical diagnosis [56, 57].
For example, in the European Sleep Apnoea Database (ESADA) cohort of over 25000 patients living in Europe, a study of phenotypes in 6500 patients revealed that over half of patients with OSA in the cohort had an "insomnia" phenotype and were more likely to experience cardiovascular comorbidity than the "sleepy" phenotype, despite less severe AHI [58]. Additionally, phenotypes with excessive daytime sleepiness were more likely to be CPAP adherent [58]. A further study in 23000 patients in the ESADA cohort using cluster analysis identified eight different phenotypes, incorporating four phenotypes exclusively present according to sex [59]. Although this is interesting and potentially useful, further work needs to be undertaken to validate these observations in other cohorts, with and without the use of the $\mathrm{AHI}$ as a clustering variable and in the context of sleepiness alone.

Most recently, a study using the "Baveno" classification, a multicomponent grading system for OSA [60], was tested in the ESADA cohort in 14499 patients with a diagnosis of OSAS who were on treatment [61]. Groups were classified into patients with minor symptoms and cardiometabolic comorbidities (Group A), severe symptoms and minor cardiometabolic comorbidities (Group B), minor symptoms and severe cardiometabolic comorbidities (Group C) and severe symptoms and cardiometabolic comorbidities (Group D). Symptoms were based on the ESS, and AHI was

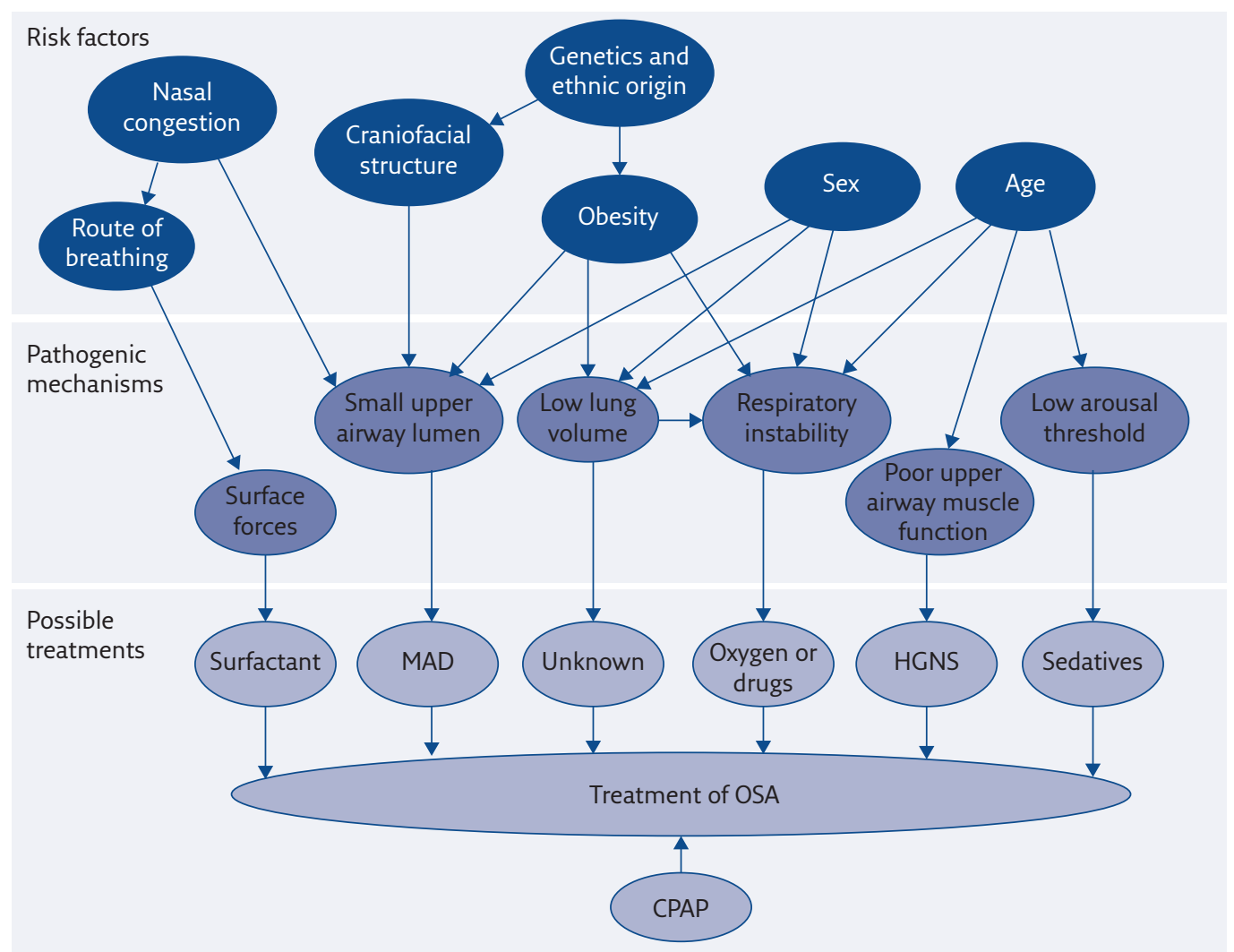

Figure 3 Schema of risk factors and mechanisms in the pathogenesis of OSA/OSAS and how they can be addressed in precision treatment. MAD: mandibular advancement device; HGNS: hypoglossal nerve stimulation. Reproduced from [54] with permission. 
also incorporated into stratification on one iteration. Approximately $70 \%$ of the patients in the entire group were on CPAP and a subgroup were followed up for 24-36 months. The major findings (apart from reasonable ease of classification) showed that Groups B, C and D improved on treatment with CPAP on several cardiometabolic parameters and that the $\mathrm{AHI}$ did not improve the classification integrity [61].

On an individual level, one very direct method of phenotyping patients is by using drug-induced sedation endoscopy (DISE) [62]. DISE takes clinical investigation one step further in allowing for the direct observation of different patterns and levels of upper airway collapse in patients under anaesthesia (usually propofol/midazolam). This is an important technique in allowing for better risk stratification regarding treatment, decisions about continuing CPAP, suitability for mandibular advancement devices or surgery. However, this is not commonly available outside of specialised ENT (ear, nose and throat) units and is unlikely to be feasible in terms of providing a general definition of the disorder which can be applied in the outpatient setting. Additionally, the drugs may affect upper airway dilator muscle activity, thereby influencing the phenomenon of interest.

Finally, as suggested by others in the past [63] and based on evidence [64], CPAP responsiveness of the diurnal symptoms and comorbidities arising from OSA could very well be a more meaningful addition to the definition of OSAS than the ones in current use.

\section{Conclusion}

At first sight, the definition of OSAS appears simple, albeit one of halves. However, it is a definition that has been in a state of flux since the 1970s and is dependent on the technical acquisition of electrophysiological signals subject to ever greater but nonstandardised precision, fluctuating scoring definitions subject to manual counting with inherent inter- and intra-scorer bias, and somewhat arbitrary definitions of sleepiness. The sleep community has further complicated matters by not adhering to strict definitions when it has come to research, and furthermore, has over-concentrated on a metric, the $\mathrm{AHI}$ (and related metrics), to define OSA primarily, rather than OSAS. This has resulted in a body of

\section{Self-evaluation questions}

1. The duration of an apnoea and hypopnoea are defined as:
a) $10 \mathrm{~s}$ minimum in duration for both events
b) $5 \mathrm{~s}$ for a hypopnoea and $10 \mathrm{~s}$ for an apnoea
c) There is no duration definition
d) $20 \mathrm{~s}$ in duration for both events
e) $10 \mathrm{~s}$ for a hypopnoea and $5 \mathrm{~s}$ for an apnoea

2. Abnormal sleepiness using the ESS is defined as:
a) A score of 11 out of 24 in Australia only
b) A score of 11 out of 24 universally
c) A score of 11 out of 24 in patients under the age of 70 years
d) A score of 5 out of 24
e) There is no absolute definition of sleepiness using the ESS

3. Apart from measuring breathing signals, the PSG can be used to measure which of the following?
a) Rapid eye movement sleep
b) Non-rapid eye movement sleep
c) Period limb movements
d) Snoring
e) All of the above

4. The definition of the OSAS requires which of the following?
a) $\mathrm{An} \mathrm{AHI}$ of 10 events per hour of sleep and no symptoms
b) The presence of apnoeas and hypopnoeas during sleep resulting in daytime sleepiness
c) Excessive daytime sleepiness due to depression
d) The presence of insomnia
e) Hypoventilation

work which has led us to a better understanding of the pathophysiology and potential consequences of OSA, but perhaps has left us with more questions and sometimes puzzling and contradictory answers. Technological advances in the physical equipment used to acquire overnight signals have almost outstripped increasingly outmoded classification systems and methods of practice and possibly our definitions of breathing events. With more sensitive tools, the validity of previous studies is called into question and new algorithms for defining OSAS are starting to emerge. Perhaps we have come full circle in that we are challenging ourselves again in how to conceptualise this important disorder, as we did in the 1970s. For the time being, as with "love", so with OSAS: everyone knows what it is, but it remains difficult to define precisely.

\section{Affiliations}

\section{Renata L. Riha ${ }^{1,2}$}

${ }^{1}$ Dept of Sleep Medicine, Royal Infirmary Edinburgh, Edinburgh, UK.

2Sleep Research Unit, Centre for Clinical Brain Sciences, University of Edinburgh, Edinburgh, UK.

\section{Conflict of interest}

R.L. Riha has nothing to disclose. 


\section{Suggested answers}

1. a.

2. b.

3. e.

4. b.

\section{References}

1. Riha RL. Diagnostic approaches to respiratory sleep disorders. J Thorac Dis 2015; 7: 1373-1384.

2. Patil SP, Ayappa IA, Caples SM, et al. Treatment of adult obstructive sleep apnea with positive airway pressure: an American Academy of Sleep Medicine clinical practice guideline. J Clin Sleep Med 2019; 15: 335-343.

3. Sutherland K, Kairaitis K, Yee BJ, et al. From CPAP to tailored therapy for obstructive sleep apnoea. Multidiscip Respir Med 2018; 13: 44

4. Malhotra A, Ayappa I, Ayas N, et al. Metrics of sleep apnea severity: beyond the AHI. Sleep 2021; 44: zsab030.

5. Pevernagie DA, Gnidovec-Strazisar B, Grote L, et al. On the rise and fall of the apnea-hypopnea index: a historical review and critical appraisal. J Sleep Res 2020; 29: e13066.

6. American Academy of Sleep Medicine. The International Classification of Sleep Disorders, Revised (ICSD-R). ISBN 0-9657220-1-5. Westchester, AASM, 2001; pp. 57-58.

7. American Academy of Sleep Medicine. The International Classification of Sleep Disorders, Second Edition (ICSD-2). ISBN 0-9657220-2-3. Westchester, AASM, 2005; p. 54.

8. American Academy of Sleep Medicine. The International Classification of Sleep Disorders, Third Edition (ICSD-3). ISBN 978-0991543410. Westchester, AASM, 2014; pp. 53-54.

9. Benjafield AV, Ayas NT, Eastwood PR, et al. Estimation of the global prevalence and burden of obstructive sleep apnoea: a literature-based analysis. Lancet Respir Med 2019; 7: 687-698.

10. Adams R, Appleton S, Taylor A, et al. Are the ICSD-3 criteria for sleep apnoea syndrome too inclusive? Lancet Respir Med 2016; 4: e19-e20.

11. Young T, Palta M, Dempsey J, et al. The occurrence of sleepdisordered breathing among middle-aged adults. N Engl J Med 1993; 328: 1230-1235.

12. Gottlieb DJ, Punjabi NM. Diagnosis and management of obstructive sleep apnea: a review. JAMA 2020; 323: 1389-1400.

13. Heinzer R, Vat $S$, Marques-Vidal P, et al. Prevalence of sleep-disordered breathing in the general population: the HypnoLaus study. Lancet Respir Med 2015; 3: 310-318.

14. Kushida CA, Nichols DA, Simon RD, et al. Symptom-based prevalence of sleep disorders in an adult primary care population. Sleep Breath 2000; 4: 9-14.

15. Ohayon MM. From wakefulness to excessive sleepiness: what we know and still need to know. Sleep Med Rev 2008; 12: 129-141.

16. Ohayon MM, Dauvilliers Y. Reynolds CF 3rd. Operational definitions and algorithms for excessive sleepiness in the general population: implications for DSM-5 nosology. Arch Gen Psychiatry 2012; 69: 71-79.

17. Chervin RD. Sleepiness, fatigue, tiredness, and lack of energy in obstructive sleep apnea. Chest 2000; 118: 372-379.

18. Baiardi S, Mondini S. Inside the clinical evaluation of sleepiness: subjective and objective tools. Sleep Breath 2020; 24: 369-377

19. Hoddes E, Zarcone V, Smythe H, et al. Quantification of sleepiness: a new approach. Psychophysiology 1973; 10 431-436.

20. Johns MW. A new method for measuring daytime sleepiness: the Epworth sleepiness scale. Sleep 1991; 14: 540-545.

21. Ghiassi R, Murphy K, Cummin AR, et al. Developing a pictorial Epworth sleepiness scale. Thorax 2011; 66: 97-100.

22. Campbell AJ, Neill AM, Scott DAR. Clinical reproducibility of the Epworth sleepiness scale for patients with suspected sleep apnea. J Clin Sleep Med 2018; 14: 791-795.

23. Taylor E, Zeng I, O'Dochartaigh C. The reliability of the Epworth sleepiness score in a sleep clinic population. J Sleep Res 2019; 28: e12687.

24. Stradling JR, Smith D, Crosby J. Post-CPAP sleepiness - a specific syndrome? J Sleep Res 2007; 16: 436-438.

25. Stroe AF, Roth T, Jefferson C, et al. Comparative levels of excessive daytime sleepiness in common medical disorders. Sleep Med 2010; 11: 890-896
26. Chung F, Abdullah HR, Liao P. STOP-Bang questionnaire: a practical approach to screen for obstructive sleep apnea. Chest 2016; 149: 631-638.

27. Senaratna CV, Perret JL, Matheson MC, et al. Validity of the Berlin questionnaire in detecting obstructive sleep apnea: a systematic review and meta-analysis. Sleep Med Rev 2017; 36: $116-124$

28. Herschmann S, Berger M, Haba-Rubio J, et al. Comparison of NoSAS score with Berlin and STOP-BANG scores for sleep apnea detection in a clinical sample. Sleep Med 2021; 79 : 113-116.

29. Riha RL, Staats R. "It is better to know some of the questions than all of the answers". The diagnosis of the Obstructive Sleep Apnea/Hypopnea Syndrome by questionnaires. Pulmonology 2019; 25: 134-136.

30. Fedson AC, Pack Al, Gislason T. Frequently used sleep questionnaires in epidemiological and genetic research for obstructive sleep apnea: a review. Sleep Med Rev 2012; 16 : 529-537.

31. Bonsignore MR, Randerath W, Schiza S, et al. European Respiratory Society statement on sleep apnoea, sleepiness and driving risk. Eur RespirJ 2021; 57: 2001272.

32. van der Heide A, van Schie MK, Lammers GJ, et al. Comparing treatment effect measurements in narcolepsy: the sustained attention to response task. Epworth sleepiness scale and maintenance of wakefulness test. Sleep 2015; 38: 1051-1058.

33. Huang Y, Aumüller P, Fietze I, et al. Comparison of the Oxford sleep resistance test and the multiple sleep latency test. Physiol Meas 2020; 41: 104005

34. Littner MR, Kushida C, Wise M, et al. Practice parameters for clinical use of the multiple sleep latency test and the maintenance of wakefulness test. Sleep 2005; 28 : 113-121.

35. Iber C, Ancoli-Israel S, Chesson A, et al. The AASM Manual for the Scoring of Sleep and Associated Events: Rules, Terminology and Technical Specifications. Westchester, American Academy of Sleep Medicine, 2007.

36. Hirotsu C, Haba-Rubio J, Andries D, et al. Effect of three hypopnea scoring criteria on OSA prevalence and associated comorbidities in the general population. J Clin Sleep Med 2019; 15: 183-194.

37. Duce B, Milosavljevic J, Hukins C. The 2012 AASM respiratory event criteria increase the incidence of hypopneas in an adult sleep center population. J Clin Sleep Med 2015; 11: 1425-1431.

38. Ruehland WR, Rochford PD, O'Donoghue FJ, et al. The new AASM criteria for scoring hypopneas: impact on the apnea hypopnea index. Sleep 2009; 32: 150-157.

39. Meyer TJ, Eveloff SE, Kline LR, et al. One negative polysomnogram does not exclude obstructive sleep apnea. Chest 1993: 103: 756-760.

40. Shayeb M E, Topfer LA, Stafinski T, et al. Diagnostic accuracy of level 3 portable sleep tests versus level 1 polysomnography for sleep-disordered breathing: a systematic review and metaanalysis. CMAJ 2014; 186: E25-E51.

41. Escourrou P, Grote L, Penzel T, et al. The diagnostic method has a strong influence on classification of obstructive sleep apnea. J Sleep Res 2015; 24: 730-738.

42. Flemons WW, Littner MR, Rowley JA, et al. Home diagnosis of sleep apnea: a systematic review of the literature. An evidence review cosponsored by the American Academy of Sleep Medicine, the American College of Chest Physicians, and the American Thoracic Society. Chest 2003; 124: 1543-1579.

43. Ramsey R, Mehra R, Strohl KP. Variations in physician interpretation of overnight pulse oximetry monitoring. Chest 2007; 132: 852-859.

44. Collop NA, Tracy SL, Kapur V, et al. Obstructive sleep apnea devices for out-of-center $(\mathrm{OOC})$ testing: technology evaluation. J Clin Sleep Med 2011; 7: 531-548.

45. Casey KR, Tiwari R. The (still) elusive definition of hypopnea. J Clin Sleep Med 2018; 14: 1971-1972. 
46. Sleep-related breathing disorders in adults: recommendations for syndrome definition and measurement techniques in clinical research. The report of an American Academy of Sleep Medicine task force. Sleep 1999; 22: 667-689.

47. Kingshott RN, Douglas NJ. The effect of in-laboratory polysomnography on sleep and objective daytime sleepiness. Sleep 2000; 23: 1109-1113.

48. Tkacova R, McNicholas WT, Javorsky M, et al. Nocturnal intermittent hypoxia predicts prevalent hypertension in the European Sleep Apnoea Database cohort study. Eur Respir J 2014; 44: 931-941.

49. O'Mahony AM, Garvey JF, McNicholas WT. Technologic advances in the assessment and management of obstructive sleep apnoea beyond the apnoea-hypopnoea index: a narrative review. J Thorac Dis 2020; 12: 5020-5038.

50. Bonsignore MR, Saaresranta T, Riha RL. Sex differences in obstructive sleep apnoea. Eur Respir Rev 2019; 28: 190030.

51. Kohler M, Bloch KE, Stradling JR. The role of the nose in the pathogenesis of obstructive sleep apnea. Curr Opin Otolaryngol Head Neck Surg 2009; 17: 33-37.

52. Riha RL, Kotoulas SC, Pataka A, et al. Obstructive sleep apnoea in adult patients post-tonsillectomy. Sleep Med 2021; 78: 189-192.

53. Eckert DJ. Phenotypic approaches to obstructive sleep apnoea - new pathways for targeted therapy. Sleep Med Rev 2018; 37: 45-59.

54. Jordan AS, McSharry DG, Malhotra A. Adult obstructive sleep apnoea. Lancet 2014; 383: 736-747.

55. Dutta R, Delaney G, Toson B, et al. A novel model to estimate key obstructive sleep apnea endotypes from standard polysomnography and clinical data and their contribution to obstructive sleep apnea severity. Ann Am Thorac Soc 2021; 18: 656-667.

56. Martinez-Garcia MA, Campos-Rodriguez F, Barbé F, et al. Precision medicine in obstructive sleep apnoea. Lancet Respir Med 2019; 7: 456-464.

57. Pien GW, Ye L, Keenan BT, et al. Changing faces of obstructive sleep apnea: treatment effects by cluster designation in the Icelandic sleep apnea cohort. Sleep 2018; 41: zsx201.

58. Saaresranta T, Hedner J, Bonsignore MR, et al. Clinical phenotypes and comorbidity in European sleep apnoea patients. PLoS One 2016; 11: e0163439.

59. Bailly S, Grote L, Hedner J, et al. Clusters of sleep apnoea phenotypes: a large pan-European study from the European Sleep Apnoea Database (ESADA). Respirology 2021; 26: 378-387.

60. McNicholas WT, Bassetti CL, Ferini-Strambi L, et al. Challenges in obstructive sleep apnoea. Lancet Respir Med 2018; 6: 170-172.

61. Randerath WJ, Herkenrath S, Treml M, et al. Evaluation of a multicomponent grading system for obstructive sleep apnoea: the Baveno classification. ERJ Open Res 2021; 7: 00928-2020.

62. Vroegop AV, Vanderveken OM, Verbraecken JA. Drug-induced sleep endoscopy: evaluation of a selection tool for treatment modalities for obstructive sleep apnea. Respiration 2020; 99 : 451-457

63. Stradling JR, Davies RJ. Sleep. 1: Obstructive sleep apnoea/ hypopnoea syndrome: definitions, epidemiology, and natural history. Thorax 2004; 59: 73-78.

64. Kingshott RN, Vennelle M, Hoy CJ, et al. Predictors of improvements in daytime function outcomes with CPAP therapy. Am J Respir Crit Care Med 2000; 161: 866-871. 